\title{
A LIMIT THEOREM FOR THE FRACTIONAL PARTS OF THE SEQUENCE $\left\{2^{k} t\right\}$
}

\section{Z. CIESIELSKI AND H. KESTEN}

1. Introduction. Let

$$
x_{n}(t)=\frac{1}{n^{1 / 2}} \sum_{i=0}^{n-1}\left[f_{t}\left(2^{i} x\right)-t\right]
$$

for $n=1,2, \cdots$, where

$$
f_{t}(x)= \begin{cases}1 & \text { for } 0<x<t \\ 0 & \text { for } t \leqq x \leqq 1\end{cases}
$$

and $f_{t}(x+1)=f_{t}(x)(0 \leqq t \leqq 1)$. For fixed $n$ we consider $\left\{x_{n}(t)\right\}$ as a stochastic process with the time interval $\langle 0,1\rangle$ and multivariate distribution defined as follows:

$$
\begin{aligned}
P\left\{x_{n}\left(t_{1}\right)<\omega_{1}, \cdots, x_{n}\left(t_{s}\right)<\omega_{s}\right\} & \\
& =m\left\{x: 0 \leqq x \leqq 1 ; x_{n}\left(t_{i}\right)<\omega_{i}, i=1, \cdots, s\right\},
\end{aligned}
$$

where $m$ denotes the Lebesgue measure.

It was proved by N. J. Fine [2] that for given $t_{i} \in\langle 0,1\rangle$ and $\omega_{i}$ ( $s$ any positive integer; for $s=1$ see [3]), the probability (1.3) approaches

$$
P\left\{x\left(t_{1}\right)<\omega_{1}, \cdots, x\left(t_{s}\right)<\omega_{s}\right\}
$$

as $n \rightarrow \infty$. Here $\{x(t)\}, 0 \leqq t \leqq 1$, is a Gaussian process associated with the covariance

$$
\rho(t, s)=\lim _{n \rightarrow \infty} \int_{0}^{1} x_{n}(t) x_{n}(s) d x
$$

and with $E\{x(t)\}=0$.

The purpose of this paper is to prove the following limit theorem, which was conjectured by M. Kac:

$$
\lim _{n \rightarrow \infty}\left\{\sup _{0 \leqq t \leqq 1}\left|x_{n}(t)\right|<\omega\right\}=P\left\{\max _{0 \leqq t \leqq 1}|x(t)|<\omega\right\} .
$$

Actually we prove convergence in distribution for a wide class of functionals of the sample functions by using a general invariance theorem of Skorokhod [5]. $\sup _{0 \leq t \leq 1}(\cdot)$ is only a specific example of the result.

Received by the editors June 1, 1961. 
2. Preliminary results. In our proof we shall use the following results.

Let ${ }^{1} K$ be the space of all left continuous functions on $\langle 0,1\rangle$ which also have right limits at every point and are right continuous at zero.

The topology $J$ in $K$ is determined by the following convergence: the sequence $y_{n}(t)$ converges to $y_{0}(t)$ if there exists a sequence of monotonic continuous functions $\lambda_{n}(t)$ for which

$$
\begin{gathered}
\lambda_{n}(0)=0, \quad \lambda_{n}(1)=1, \quad \lim _{n \rightarrow \infty} \max _{0 \leqq t \leqq 1}\left|\lambda_{n}(t)-t\right|=0, \\
\lim _{n \rightarrow \infty} \sup _{0 \leqq t \leqq 1}\left|y_{n}\left(\lambda_{n}(t)\right)-y_{0}(t)\right|=0 .
\end{gathered}
$$

Lemma $1,[5]$. Let $\left\{\xi_{n}(t)\right\}, 0 \leqq t \leqq 1, n=0,1, \cdots$, be a sequence of stochastic processes such that $\xi_{n}(t), n=0,1, \cdots$, are in $K$ with probability one. Then, the distribution of $F\left(\xi_{n}\right)$ approaches the distribution of $F\left(\xi_{0}\right)$ for every real functional $F(\cdot)$, defined on $K$, and continuous in the topology $J$, if and only if

(a) The multivariate distribution of $\left[\xi_{n}\left(t_{1}\right), \cdots, \xi_{n}\left(t_{s}\right)\right]$ approaches the multivariate distribution of $\left[\xi_{0}\left(t_{1}\right), \cdots, \xi_{0}\left(t_{s}\right)\right]$ for all $s$, and $t_{1}, \cdots, t_{s}$ from some set $N$ which is dense in $\langle 0,1\rangle$,

(b) for each $\epsilon>0$

$\lim _{c \downarrow 0} \lim _{n \rightarrow \infty} P\left\{\sup _{t-c<t_{1}<t<t_{2}<t+c} \min \left[\left|\xi_{n}\left(t_{1}\right)-\xi_{n}(t)\right|,\left|\xi_{n}(t)-\xi_{n}\left(t_{2}\right)\right|\right]>\epsilon\right\}$

$$
=0 \text {. }
$$

Lemma 2 (S. Bernstein, see [4, p. 19]). Let $\left\{Y_{k}\right\}, k=1, \cdots, n$, be independent binomial random variables such that $P\left\{Y_{k}=1\right\}=p$, $P\left\{Y_{k}=0\right\}=q$. Then

$$
P\left\{\left|\sum_{k=1}^{n}\left(Y_{k}-p\right)\right| \geqq 2 \omega(n p q)^{1 / 2}\right\} \leqq 2 e^{-\omega^{2}}
$$

for $0 \leqq \omega \leqq(3 / 2)(n p q)^{1 / 2}$.

LEMMA 3. Let $x_{n}(t)$ be defined by (1.1) and (1.2) and let $n>m \geqq 0$, $1 \leqq i \leqq 2^{m}$. Then for $0 \leqq \omega \leqq 3(n-m) / 2^{m+1 / 2} n^{1 / 2}$

$$
P\left\{\left|x_{n}\left(\frac{i}{2^{m}}\right)-x_{n}\left(\frac{i-1}{2^{m}}\right)\right|>\omega\right\} \leqq 2 m \exp \left(-\frac{\omega^{2} 2^{m}}{8(m+1)}\right) .
$$

Proof. We can assume $m \geqq 1$ because for $m=0$ the inequality is

1 We take left continuity instead of right continuity as required by Skorokhod [5]. This clearly does not change the validity of Skorokhod's result but is somewhat more convenient for the proof of Lemma 3 . 
trivial. Let $I=\left\langle i / 2^{m},(i-1) / 2^{m}\right\rangle$, let $x$ have a uniform distribution on $\langle 0,1\rangle$, and let

$$
Y_{k}=Y_{k}(x)= \begin{cases}1 & \text { if } 2^{k} x(\bmod 1) \in I \\ 0 & \text { if } 2^{k} x(\bmod 1) \notin I\end{cases}
$$

If $x$ has the diadic expansion

$$
x=\sum_{r=1}^{\infty} \epsilon_{r}(x) / 2^{r}
$$

then

$$
2^{k} x(\bmod 1)=\sum_{r=k+1}^{\infty} 2^{k-r} \epsilon_{r}(x)
$$

and $Y_{k}$ depends only on the digits $\epsilon_{k+1}, \cdots, \epsilon_{k+m}$. Thus, for every $k, Y_{k}, Y_{k+m}, Y_{k+2 m}, \cdots$ are independent binomial variables with probability of success $p=2^{-m}$. By Lemma 2 we get

$$
P\left\{\left|\sum_{s=0}^{r}\left(Y_{m s+k}-p\right)\right|>2 \omega((r+1) p)^{1 / 2}\right\} \leqq 2 e^{-\omega^{2}}
$$

for $0 \leqq \omega \leqq\left(3 / 2^{3 / 2}\right)((r+1) p)^{1 / 2}$. If we apply (2.3) with $r=r_{k}=[n / m]$ for $k \leqq n-m[n / m]$ and $r=r_{k}=[n / m]-1$ for $k>n-m[n / m]$ (here $[x]$ denotes the greatest integer $\leqq x)$ we get

$$
\begin{aligned}
P\left\{\left|\sum_{k=1}^{n}\left(Y_{k}-p\right)\right|>\omega\right\} & \leqq \sum_{k=1}^{m} P\left\{\left|\sum_{\varepsilon=0}^{r_{k}}\left(Y_{m s+k}-p\right)\right|>\frac{\omega}{m}\right\} \\
& \leqq 2 m \exp \left(-\frac{\omega^{2}}{4 m(n+m) p}\right)
\end{aligned}
$$

for $0 \leqq \omega \leqq\left(3 / 2^{1 / 2}\right)(p(n-m))$. Finally, according to $(2.2)$ we have

$$
x_{n}\left(\frac{i}{2^{m}}\right)-x_{n}\left(\frac{i-1}{2^{m}}\right)=\frac{1}{n^{1 / 2}} \sum_{k=1}^{n}\left(Y_{k}-p\right),
$$

hence our lemma follows.

3. The proof of (1.6). Let us put in Lemma $1 \xi_{n}(t)=x_{n}(t)$, $n=1,2, \cdots$, and $\xi_{0}(t)=x(t)$. It is clear that $x_{n}(t)$ according to (1.1) and (1.2) is left continuous (in $t$ ), has right hand limits and is right continuous at zero. It was also proved in [1] that $x(t)$ is continuous with probability one. Condition (a) of Lemma 1 is satisfied by Fine's result [2]. Thus, if we show that condition (b) is also satisfied then 
the convergence in distribution for any functional $F$ on $K$, continuous in the topology $J$, follows from Lemma 1 . Clearly the functional

$$
F_{0}(y)=\sup _{0 \leqq t \leqq 1}|y(t)|, \quad y(\cdot) \in K
$$

is continuous in the topology $J$ (cf. (2.1)). We may write max instead of sup in the right hand side of (1.6) because $x(t)$ is continuous with probability one.

Let now $M$ be fixed integer and put

$$
N=\left[\frac{2}{3} \log _{2} n\right] \text {. }
$$

Let $1 \leqq k \leqq 2^{N}, 1 \leqq u \leqq 2^{M}$ and

$$
\frac{u-1}{2^{M}} \leqq \frac{k}{2^{N}}<\frac{u}{2^{M}}
$$

We can then find $\eta(M+1), \eta(M+2), \cdots, \eta(N), \eta=0,1$ such that

$$
\frac{k}{2^{N}}=\frac{u-1}{2^{M}}+\sum_{m=M+1}^{N} \frac{\eta(m)}{2^{m}} \text {. }
$$

Consequently, if we define $u_{m}$ by

$$
u_{m}=\frac{u-1}{2^{M}}+\sum_{j=M+1}^{m} \frac{\eta(j)}{2^{j}}, \quad u_{M}=\frac{u-1}{2^{M}}
$$

then

$$
\begin{aligned}
\left|x_{n}\left(\frac{k}{2^{N}}\right)-x_{n}\left(\frac{u-1}{2^{M}}\right)\right| & \leqq \sum_{m=M+1}^{N}\left|x_{n}\left(u_{m-1}\right)-x_{n}\left(u_{m-1}+\frac{1}{2^{m}}\right)\right| \\
& \leqq \sum_{m=M+1}^{N} \max _{1 \leqq i \leqq 2 m}\left|x_{n}\left(\frac{i}{2^{m}}\right)-x_{n}\left(\frac{i-1}{2^{m}}\right)\right| .
\end{aligned}
$$

Moreover, for $(k-1) / 2^{N} \leqq t<k / 2^{N}$, by (1.1) and (1.2)

$$
\begin{aligned}
\left|x_{n}(t)-x_{n}\left(\frac{k-1}{2^{N}}\right)\right| & \leqq\left|x_{n}\left(\frac{k}{2^{N}}\right)-x_{n}\left(\frac{k-1}{2^{N}}\right)\right|+\frac{n^{1 / 2}}{2^{N}} \\
& \leqq\left|x_{n}\left(\frac{k}{2^{N}}\right)-x_{n}\left(\frac{k-1}{2^{N}}\right)\right|+2 n^{-1 / 6}
\end{aligned}
$$

Combining (3.1)-(3.3), we have 


$$
\begin{aligned}
& \sup _{\left|t_{1}-t_{i}\right| \leqq 2^{-M-1}}\left|x_{n}\left(t_{1}\right)-x_{n}\left(t_{2}\right)\right| \\
& \quad \leqq 4 n^{-1 / 6}+4 \sum_{m=M}^{N} \max _{1 \leqq i \leqq 2^{m}}\left|x_{n}\left(\frac{i}{2^{m}}\right)-x_{n}\left(\frac{i-1}{2^{m}}\right)\right| .
\end{aligned}
$$

However, by Lemma 3 , we have for any $0 \leqq \omega \leqq 3 / 2 \cdot 2^{1 / 2}$ and $m \leqq 2 \log _{2} n / 3$

$$
\begin{aligned}
P\left\{\max _{1 \leqq i \leqq 2^{m}}\left|x_{n}\left(\frac{i}{2^{m}}\right)-x_{n}\left(\frac{i-1}{2^{m}}\right)\right|>\omega 2^{-m / 4}\right\} & \\
& \leqq 2^{m+1} m \exp \left(-\frac{\omega^{2} 2^{m / 2}}{8(m+1)}\right)
\end{aligned}
$$

and therefore

$$
\sum_{m=M}^{N} \max _{1 \leqq i \leqq 2^{m}}\left|x_{n}\left(\frac{i}{2^{m}}\right)-x_{n}\left(\frac{i-1}{2^{m}}\right)\right| \leqq \omega \sum_{m=M}^{N} 2^{-m / 4}
$$

except for a set of probability not exceeding

$$
\sum_{m=M}^{\infty} 2^{m+1} m \exp \left(-\frac{\omega^{2} 2^{m / 2}}{8(m+1)}\right) .
$$

Since both (3.6) and the right hand side of (3.5) tend to zero as $M \rightarrow \infty$, condition (b) follows from (3.4). This completes the proof.

\section{REFERENCES}

1. Z. Ciesielski, Hölder conditions for realizations of Gaussian processes, Trans. Amer. Math. Soc. 99 (1961), 403-413.

2. N. J. Fine, On the asymptotic distributions of certain sums, Proc. Amer. Math. Soc. 5 (1954), 243-252.

3. M. Kac, On the distributions of values of sums of type $\sum f\left(2^{k} t\right)$, Ann. of Math. 47 (1946), 33-49.

4. G. G. Lorenz, Bernstein polynomials, Toronto, 1953.

5. A. V. Skorokhod, Limit theorems for stochastic processes, Theory of Probability and its Applications 1 (1956), 261-290 (An English translation of the Soviet journal, Teor. Veroyatnost. i. Primenen.)

Cornell University and

HEBREW UNIVERSITY, JeRUSALEM, ISRAEL 